NBI-HE-95-34

October, 1995

\title{
OPE formulae for deformed super-Virasoro algebras
}

\author{
Haru-Tada Sato 円 \\ The Niels Bohr Institute, University of Copenhagen \\ Blegdamsvej 17, DK-2100 Copenhagen, Denmark
}

\begin{abstract}
We show the OPE formulae for three types of deformed super-Virasoro algebras: Chaichian-Presnajder's deformation, Belov-Chaltikhian's one and its modified version. Fundamental (anti-)commutation relations toward a ghost realization of deformed super-Virasoro algebra are also discussed.
\end{abstract}

\footnotetext{
${ }^{1}$ Fellow of Danish Research Academy

E-mail address : sato@nbivax.nbi.dk
} 


\section{Introduction}

In this paper, we analyze the deformed super-Virasoro superalgebras, based on the Sugawara construction, proposed in recent years [1], [2] It might be useful for generalizing conformal field and string theories to investigate the deformed super-Virasoro algebras, which are different from $W$-infinity algebras in the sense of including an independent parameter, namely, a deformation parameter. For example, in conformal field theories, exactly speaking in the thermodynamic limit of the XYZ Heisenberg chain model [6], there exists a deformed Virasoro algebra [3], [4], [5] which satisfies quadratic relations related to the Zamolodchikov-Faddeev algebra for deformed chiral vertex operators. The algebra of [1] corresponds to a particular case of this case [3]. As for deformation of string theories, it is interesting to analyze the deformed Virasoro algebra on a speculative ground that the Virasoro constraints would lead to deformation of the BRS operator, conformal anomalies, and thus to the critical dimensions depending on a deformation parameter $q$. The $q$ might be related to something like an energy scale where the spacetime dimension changes.

There are two types of the deformed Virasoro algebras which are made by the Sugawara construction, namely in terms of the bosonic and fermionic Heisenberg oscillators [1]. Each possesses different structure constants but reduces to the same Virasoro algebra in $q \rightarrow 1$ up to the difference of central charges. We refer to the bosonic realization as $q$ - $\operatorname{Vir}^{B}$ and to the fermionic one as $q$ - $\operatorname{Vir}^{F}$ for convenience (this naming follows from [2]). Further, Chaichian and Presnajder introduced a deformed spin-3/2 current and proposed a deformed supersymmetric Virasoro algebra which is constituted of $q$-Vir ${ }^{F}, q$-Vir ${ }^{B}$ and the deformed superconformal current. However much inconvenience will be raised by the difference of structure constants between bosonic and fermionic algebras in: anomaly cancellation, superfield formulation, deformation of the ghost Virasoro and BRS algebras.

Belov and Chaltikhian improved the unpleasant feature of the above deformed superVirasoro algebra and proposed a supersymmetric $q$-Vir ${ }^{B}$ algebra. They modified the basis of the fermionic Heisenberg algebra in order that the fermionic $q$-Virasoro generators 
satisfy the same commutation relation as $q-\mathrm{Vir}^{B}$. This achievement means that the fermionic and bosonic sectors can be organized into unique commutation relation or operator product expansion (OPE), which is convenient to construct BRS operator and to discuss other algebraic structure. We should furthermore find ghost realization of the deformed Virasoro algebra in order to make a progress in deformed string theories. To find a complete supersymmetric deformed Virasoro algebra, it is thus useful to list up OPE formulae for several types of supersymmetric deformed Virasoro algebra.

In sect. 2, we review Chaichian-Presnajder's superalgebra adding brief comments on the Ramond sector. Then, we provide the OPE formulae for this superalgebra. In sect. 3, we are concerned with Belov-Chaltikhian's superalgebra in which they have not shown any (anti-)commutators other than one of (anti-)commutation relations. We

first complete all (anti-)commutators including central extensions for this superalgebra and then exhibit the OPE formulae for them. Simplification of this superalgebra is also presented. In sect. 4 , we intend to discuss the deformed Virasoro algebras in terms of $b c$ system. New type of deformed Virasoro algebra, a realization of $q$-Vir ${ }^{F}$ and basic set of algebras toward a $q$-Virasoro superalgebra are shown. Conclusion is in sect. 5 .

\section{Chaichian-Presnajder's superalgebra}

Let us first explain some notations which make expressions simple. We introduce the following $q$-brackets for an arbitrary rational number $x$ :

$$
[x]=\frac{q^{x}-q^{-x}}{q-q^{-1}} \quad[x]_{ \pm}=\left(q^{x} \pm q^{-x}\right) / 2,
$$

where $q$ is not a root of unity to avoid $[x]=0$ for any $x$. The $q$-difference of $f(z)$ at $z=a$ is

$$
\partial^{q} f(a)=\left.\partial_{z}^{q} f(z)\right|_{z=a}=\left.\frac{f(z q)-f\left(z q^{-1}\right)}{z\left(q-q^{-1}\right)}\right|_{z=a}
$$

and note that the difference $\partial^{q} f(z a) \neq \partial_{z}^{q} f(z a)$. It is also convenient to use

$$
(z-w)_{q}^{2}=(z-q w)\left(z-q^{-1} w\right)
$$


Finally, we often use the abbreviation for two letters $k$ and $l$ for simplicity

$$
Q=q^{k / 2} \quad \text { and } \quad P=q^{l / 2} .
$$

Chaichian and Presnajder proposed the following deformation of the $N=1$ superVirasoro algebra [四]:

$$
\begin{aligned}
{[F(n, k), F(m, l)] } & =\sum_{\epsilon= \pm 1} \frac{[(n \epsilon l-m k) / 2][k+\epsilon l]}{[k][\epsilon l]} F(n+m, k+\epsilon l)+C^{F}(n \mid k, l) \delta_{n+m, 0} \\
{[B(n, k), B(m, l)]=} & \frac{1}{2} \sum_{\epsilon, \eta= \pm 1}\left[\frac{n(\eta l+1)-m(\epsilon \eta k+1)}{2}\right] B(n+m, \epsilon k+l+\eta)+C^{B}(n \mid k, l) \delta_{n+m, 0} \\
{[F(n, k), G(r, l)]=} & \frac{1}{[k]\left(q-q^{-1}\right)} \sum_{\epsilon= \pm 1} \epsilon q^{(n l-\epsilon k r) / 2} G(n+r, l+\epsilon k) \\
{[B(n, k), G(r, l)]=} & \frac{-1}{2\left(q-q^{-1}\right)} \sum_{\epsilon, \eta= \pm 1} \eta q^{\frac{r(\epsilon k+\eta)-n(1+\eta)}{2}} G(n+r, \epsilon k+l+\eta) \\
\{G(r, k), G(s, l)\}= & C^{G}(r \mid l, k) \delta_{r+s, 0}+2 q^{(r l+s k) / 2} B(r+s, k-l) \\
& +\sum_{\epsilon= \pm 1} \epsilon q^{\frac{r(\epsilon-l)-s(k+\epsilon)}{2}}[k-l+\epsilon] F(r+s, k-l+\epsilon)
\end{aligned}
$$

where

$$
\begin{aligned}
C^{F}(n \mid k, l) & =\frac{1}{2[k][l]} \sum_{m=1}^{n}\left[\left(\frac{n+1}{2}-m\right) k\right]\left[\left(\frac{n+1}{2}-m\right) l\right] \\
C^{B}(n \mid k, l) & =\frac{1}{2} \sum_{m=1}^{n}\left[\left(\frac{n}{2}-m\right) k\right]_{+}\left[\left(\frac{n}{2}-m\right) l\right]_{+}[m][n-m] \\
C^{G}(r \mid l, k) & =\sum_{m=1}^{r+\frac{1}{2}} q^{\left(m-\frac{r+1}{2}\right)(l-k)}\left[r+\frac{1}{2}-m\right]=C^{G}(-r \mid k, l) .
\end{aligned}
$$

This set of commutators reduces to the usual $N=1$ super-Virasoro algebra in $q \rightarrow 1$. Eq.(2.5) is called $q$ - $\operatorname{Vir}^{F}$ and (2.6) is $q$ - $\operatorname{Vir}^{B}$ in the reference [2] for the reason that they are realized by the following Sugawara constructions

$$
\begin{aligned}
& F(n, k)=\frac{1}{2[k]} \sum_{r \in Z+1 / 2}\left[\left(\frac{n}{2}-r\right) k\right]: b_{r} b_{n-r}: \\
& B(n, k)=\frac{1}{2} \sum_{m \in Z}\left[\left(\frac{n}{2}-m\right) k\right]_{+}: a_{m} a_{n-m}:
\end{aligned}
$$




$$
G(r, k)=\sum_{m \in Z} q^{k(m-r / 2)}: a_{m} b_{r-m}:
$$

with the deformed boson $a_{n}$ and the Neveu-Schwarz fermion $b_{r}$ defined by

$$
\left[a_{n}, a_{m}\right]=[n] \delta_{n+m, 0}, \quad\left\{b_{r}, b_{s}\right\}=\delta_{r+s, 0}
$$

Note that $F$ and $B$ are symmetric under $k \rightarrow-k$ but $G$ is not. The realizations of $q$-Vir $F$ and $q-\operatorname{Vir}^{B}$ in terms of differential operators are given in [10] and [11]. On the other hand for the supercurrent $G$, it has not been given.

Before going over the OPE formulae for the above algebra, let us notice on the Ramond sector. For the Ramond fermion, we have only to change the half-integer index $r$ stemming from the fermion into integer one. Using

$$
\begin{aligned}
& {\left[F(n, k), b_{m}\right]=-\frac{1}{[k]}\left[\left(\frac{n}{2}+m\right) k\right] b_{n+m}, \quad\left[B(n, k), a_{m}\right]=-[m]\left[\left(\frac{n}{2}+m\right) k\right]_{+} a_{n+m}} \\
& \left\{G(n, k), b_{m}\right\}=q^{\left(\frac{n}{2}+m\right) k} a_{n+m}, \quad\left[G(n, k), a_{m}\right]=-[m] q^{-\left(\frac{n}{2}+m\right) k} b_{n+m},
\end{aligned}
$$

we can check that the same commutation relations as (2.5)-(2.9) are satisfied with the central extensions $C^{F}$ and $C^{G}$ replaced with

$$
\begin{gathered}
C_{R}^{F}(n \mid k, l)=\frac{1}{2[k][l]} \sum_{m=1}^{n}\left[\left(\frac{n}{2}-m\right) k\right]\left[\left(\frac{n}{2}-m\right) l\right] \\
C_{R}^{G}(n \mid l, k)=\frac{1}{2} q^{-n(l-k) / 2}[n]+\sum_{m=1}^{n} q^{\left(m-\frac{n}{2}\right)(l-k)}[n-m]=C_{R}^{G}(-n \mid k, l) .
\end{gathered}
$$

The difference between two sectors is only constant shift between the zero mode of $F$ generators

$$
F(n, k)_{N S}=F(n, k)_{R}+\frac{[k / 4]^{2}}{2[k][k / 2]} \delta_{n, 0} .
$$

We accordingly concentrate on the NS sector in the following argument.

Now we incorporate the above generators into the following composite fields as the Fourier mode operators:

$$
\begin{aligned}
& F(z ; k)=\sum_{n} F(n, k) z^{-n-2}=\frac{-1}{Q+Q^{-1}}: \psi\left(z Q^{-1}\right) \partial^{Q} \psi(z): \\
& B(z ; k)=\sum_{n} B(n, k) z^{-n-2}=\frac{1}{2}: \Phi(z Q) \Phi\left(z Q^{-1}\right):
\end{aligned}
$$




$$
G(z ; k)=\sum_{r} G(r, k) z^{-r-3 / 2}=Q^{-1 / 2}: \psi(z Q) \Phi\left(z Q^{-1}\right):
$$

where

$$
\Phi(z)=\sum_{n} a_{n} z^{-n-1}, \quad \psi(z)=\sum_{r} b_{r} z^{-r-1 / 2} .
$$

Singular parts of these bosonic and fermionic fields are expressed as

$$
\psi(z) \psi(w)=\frac{1}{z-w}, \quad \Phi(z) \Phi(w)=\frac{1}{(z-w)_{q}^{2}}
$$

and then

$$
\begin{gathered}
G(z ; k) \psi(w)=\frac{Q^{-1 / 2}}{z Q-w} \Phi\left(w Q^{-2}\right) \\
G(z ; k) \Phi(w)=Q^{-1 / 2} \partial_{w}^{q}\left(\frac{\psi\left(w Q^{2}\right)}{z Q^{-1}-w}\right) \\
F(z ; k) \psi(w)=\frac{1}{Q+Q^{-1}}\left\{\frac{\psi(w)}{(z-w)_{Q}^{2}}+\frac{\partial^{Q} \psi(w Q)}{z Q^{-1}-w}+\frac{\partial^{Q} \psi\left(w Q^{-1}\right)}{z Q-w}\right\} \\
B(z ; k) \Phi(w)=\frac{1}{2}\left\{\frac{\Phi\left(w Q^{-2} q^{-1}\right)}{(z Q-w)_{q}^{2}}+\frac{Q^{-2} \partial^{q} \Phi\left(w Q^{-2}\right)}{z Q-w q}+\quad(k \rightarrow-k)\right\} .
\end{gathered}
$$

With these OPE formulae, we obtain the OPEs corresponding to (2.5)-(2.9) up to regular terms

$$
\begin{aligned}
F(z ; k) F(w ; l)= & \frac{1}{\left(Q+Q^{-1}\right)\left(P+P^{-1}\right)(z-w P)_{Q}^{2}\left(z-w P^{-1}\right)_{Q}^{2}} \\
& +\frac{1}{w\left(q-q^{-1}\right)} \sum_{\epsilon, \eta= \pm 1} \frac{[\epsilon k+\eta l]}{[\epsilon k][\eta l]} \frac{P^{-\eta}}{z Q^{-\epsilon}-w P^{\eta}} F\left(w Q^{\epsilon} ; \epsilon k+\eta l\right) \\
B(z ; k) B(w ; l)= & \sum_{\nu= \pm 1} \frac{1 / 4}{\left(z Q-w P^{\nu}\right)_{q}^{2}\left(z Q^{-1}-w P^{-\nu}\right)_{q}^{2}} \\
& +\frac{1}{w\left(q-q^{-1}\right)} \sum_{\epsilon, \eta, \nu= \pm 1} \frac{\eta P^{-\nu} / 2}{z Q^{-\epsilon}-w P^{\nu} q^{\eta}} B\left(w Q^{\epsilon} q^{\eta / 2} ; \epsilon k+\nu l+\eta\right) \\
F(z ; k) G(w ; l)= & \frac{P^{-1}}{w[k]\left(q-q^{-1}\right)} \sum_{\epsilon= \pm 1} \frac{\epsilon Q^{\epsilon / 2}}{z-w Q^{\epsilon}} G\left(w Q^{\epsilon} ; \epsilon k+l\right) \\
B(z ; k) G(w ; l)= & \frac{P}{2 w\left(q-q^{-1}\right)} \sum_{\epsilon, \eta= \pm 1} \frac{\eta\left(Q^{\epsilon} q^{\eta / 2}\right)^{-1 / 2}}{z Q^{-\epsilon}-w P^{-1} q^{\eta}} G\left(w Q^{\epsilon} q^{\eta / 2} ; l-\epsilon k-\eta\right) \\
G(z ; k) G(w ; l)= & \frac{(P Q)^{-1 / 2}}{(z Q-w P)\left(z Q^{-1}-w P^{-1}\right)_{q}^{2}}+\frac{2(P Q)^{-1 / 2}}{z Q-w P} B\left(w Q^{-1} ; l-k\right)
\end{aligned}
$$




$$
+\sum_{\epsilon} \frac{\epsilon\left(P Q q^{\epsilon}\right)^{1 / 2}}{z Q^{-1}-w P^{-1} q^{\epsilon}}[k-l+\epsilon] F\left(w Q q^{\epsilon / 2} ; k-l+\epsilon\right) .
$$

(2.31) and (2.32) coincide with those in refs.[7] and [8]. (2.33)-(2.35) are checked by evaluating

$$
\begin{array}{r}
{[F(n, k), G(r, l)]=\frac{1}{(2 \pi i)^{2}} \oint_{0} d w \oint_{P} d z z^{n+1} w^{r+1 / 2} F(z, k) G(w, l)} \\
{[B(n, k), G(r, l)]=\frac{1}{(2 \pi i)^{2}} \oint_{0} d w \oint_{P} d z z^{n+1} w^{r+1 / 2} B(z, k) G(w, l)} \\
{[G(r, k), G(s, l)]=\frac{1}{(2 \pi i)^{2}} \oint_{0} d w \oint_{P} d z z^{r+1 / 2} w^{r+1 / 2} G(z, k) G(w, l),}
\end{array}
$$

where $\oint_{P}$ counts contribution from all poles in $z$ plane. In appendix, we show an alternative representation of the OPEs written in (anti-)commutator forms.

\section{Belov-Chaltikhian's superalgebra}

In this section, we choose different normalization for the fermionic Heisenberg algebra

$$
\left\{d_{r}, d_{s}\right\}=[r]_{+} \delta_{r+s, 0}
$$

Belov and Chaltikhian showed that

$$
H(n, k)=\frac{1}{2} \sum_{r}\left[k\left(\frac{n}{2}-r\right)\right]: d_{r} d_{n-r}:
$$

satisfies the $q$ - $\operatorname{Vir}^{B}$ algebra up to a central term for the NS fermion and discussed a supersymmetric extension [2]. However they have shown neither central extensions nor full set of supercurrent commutators. In the following, we complete all (anti-)commutation relations with central extensions including the Ramond fermion case. After that, we show the OPE formulae for their superalgebra.

According to ref.[2], we consider the following deformation of a supercurrent

$$
G^{a}(r, k)=\sum_{m}\left[\left(\frac{r}{2}-m\right) k\right]_{a}: a_{m} d_{r-m}:, \quad(a= \pm)
$$

and the sum of bosonic and fermionic realizations of the $q$-Vir ${ }^{B}$ generators

$$
L(n, k)=H(n, k)+B(n, k)
$$


In contrast with the Chaichian-Presnajder deformation, $G^{+}$is symmetric and $G^{-}$and $H$ are antisymmetric. $L$ is no longer symmetric in $k$. Commutation relations between the deformed super-Virasoro generators and the Heisenberg oscillators are

$$
\begin{aligned}
& {\left[L(n, k), d_{m}\right]=-[m]_{+}\left[\left(\frac{n}{2}+m\right) k\right] d_{n+m}, \quad\left[L(n, k), a_{m}\right]=-[m]\left[\left(\frac{n}{2}+m\right) k\right]_{+} a_{n+m}} \\
& \left\{G^{a}(r, k), d_{s}\right\}=a[s]_{+}\left[\left(\frac{r}{2}+s\right) k\right]_{a} a_{r+s}, \quad\left[G^{a}(r, k), a_{m}\right]=-[m]\left[\left(\frac{r}{2}+m\right) k\right]_{a} d_{r+m} .
\end{aligned}
$$

With use of these formulae, we obtain the following superalgebra including central extensions

$$
\begin{aligned}
& {[L(n, k), L(m, l)]=\sum_{\epsilon, \eta= \pm 1} \frac{1}{2}\left[\frac{n(\epsilon l+1)-m(\eta k+1)}{2}\right] L(n+m, \epsilon k+\eta l+\epsilon \eta)} \\
& +\left(C^{H}(n \mid k, l)+C^{B}(n \mid k, l)\right) \delta_{n+m, 0} \\
& {\left[L(n, k), G^{a}(r, l)\right]=\frac{1}{q-q^{-1}} \sum_{\epsilon, \eta= \pm 1} \eta^{\frac{1-a}{2}}\left[\frac{n(\eta l+1)-r(\epsilon k+1)}{2}\right]_{-\epsilon} G^{a \epsilon}(n+r, \epsilon k+\eta l+1)} \\
& \left\{G^{a}(r, k), G^{b}(s, l)\right\}=\frac{1}{2} \sum_{\epsilon, \eta}(-\epsilon)^{\frac{1-a}{2}}(-\eta)^{\frac{1-b}{2}}\left[\frac{r(\eta l+a b)-s(\epsilon k+a b)}{2}\right]_{a b} L(r+s, \epsilon k+\eta l+a b) \\
& +\frac{1}{4} \sum_{\epsilon, \eta}(-\epsilon)^{\frac{1-a}{2}}(-\eta)^{\frac{1-b}{2}} \tilde{C}^{G}(s \mid \epsilon k, \eta l) \delta_{r+s, 0},
\end{aligned}
$$

where $C^{H}(n \mid k, l)$ is

$$
\begin{array}{ll}
\frac{1}{2} \sum_{m=1}^{n}\left[\left(\frac{n+1}{2}-m\right) k\right]\left[\left(\frac{n+1}{2}-m\right) l\right]\left[n-m+\frac{1}{2}\right]_{+}\left[m-\frac{1}{2}\right]_{+}, & \text {for NS } \\
\frac{1}{2} \sum_{m=1}^{n}\left[\left(\frac{n}{2}-m\right) k\right]\left[\left(\frac{n}{2}-m\right) l\right][n-m]_{+}[m]_{+} & \text {for R }
\end{array}
$$

and

$$
\tilde{C}^{G}(r \mid l, k)=\sum_{r \leq m<r+s} q^{\left(\frac{s}{2}+r-m\right) l+\left(\frac{r}{2}-m\right) k}[m-r][m]_{+}, \quad\left\{\begin{array}{ll}
m \in \mathbf{Z}+\frac{1}{2}, & \text { for NS } \\
m \in \mathbf{Z}, & \text { for R }
\end{array} .\right.
$$

We should keep in mind that the number of (anti-)commutation relations is larger than that of usual case, since we have a redundant degree of freedom expressed by the superscript $a$. 
OPEs can be calculated through the following field operators

$$
\begin{gathered}
\tilde{\psi}(z)=\sum_{r} d_{r} z^{-r-1 / 2} \\
T(z ; k)=B(z ; k)+H(z ; k) \\
G^{a}(z ; k)=\frac{1}{2} \sum_{\epsilon}(-\epsilon)^{\frac{1-a}{2}} \tilde{G}(z ; \epsilon k),
\end{gathered}
$$

where

$$
H(z ; k)=\frac{1}{z\left(q-q^{-1}\right)} \tilde{\psi}(z Q) \tilde{\psi}\left(z Q^{-1}\right)
$$

and $\tilde{G}(z ; k)$ is defined by the replacement $\psi \rightarrow \tilde{\psi}$ in eq.(2.24). Singular parts of a product of $\tilde{\psi}$ is

$$
\tilde{\psi}(z) \tilde{\psi}(w)=\frac{1}{2}\left(\frac{q^{1 / 2}}{z-w q}+\frac{q^{-1 / 2}}{z-w q^{-1}}\right)
$$

and then

$$
\begin{gathered}
\tilde{G}(z ; k) \Phi(w)=Q^{-1 / 2} \partial_{w}^{q}\left(\frac{\tilde{\psi}\left(w Q^{2}\right)}{z Q^{-1}-w}\right), \quad \tilde{G}(z ; k) \tilde{\psi}(w)=\frac{1}{2} \sum_{\epsilon} \frac{Q^{-1 / 2} q^{\epsilon / 2}}{z Q-w q^{\epsilon}} \Phi\left(w q^{\epsilon} Q^{-2}\right) \\
T(z ; k) \tilde{\psi}(w)=H(z ; k) \tilde{\psi}(w)=\frac{1}{2 w\left(q-q^{-1}\right)} \sum_{\epsilon, \nu} \frac{-\epsilon q^{\nu / 2} Q^{\epsilon}}{z Q^{\epsilon}-w q^{-\nu}} \tilde{\psi}\left(w q^{-\nu} Q^{-2 \epsilon}\right) .
\end{gathered}
$$

We finally obtain the following OPE formulae (NS sector)

$$
\begin{gathered}
T(z ; k) T(w ; l)=\frac{1}{2 w\left(q-q^{-1}\right)} \sum_{\epsilon, \nu, \eta} \eta \frac{T\left(w Q^{\epsilon} q^{\eta / 2} ; \epsilon \eta l+\nu \eta k+\epsilon \nu\right)}{\left(z Q^{-\epsilon}-w P^{\nu} q^{\eta}\right) P^{\nu}} \\
+\frac{[l / 2] / 4}{q-q^{-1}} \sum_{\epsilon, \nu} \frac{q^{\epsilon} Q-q^{\nu} Q^{-1}}{\left(z Q-w q^{\nu}\right)_{P}^{2}\left(z Q^{-1}-w q^{\epsilon}\right)_{P}^{2}}+\sum_{\nu= \pm 1} \frac{1 / 4}{\left(z Q-w P^{\nu}\right)_{q}^{2}\left(z Q^{-1}-w P^{-\nu}\right)_{q}^{2}} \\
T(z ; k) G^{a}(w ; l)=\frac{1}{2 w\left(q-q^{-1}\right)} \sum_{\epsilon, \eta, \nu} \frac{\epsilon \eta \nu^{\frac{1-a}{2}}\left(Q^{\epsilon} q^{\epsilon \eta / 2}\right)^{-1 / 2}}{\left(z Q^{-\epsilon}-w P^{\nu} q^{\eta \epsilon}\right) P^{\nu}} G^{\eta a}\left(w Q^{\epsilon} q^{\epsilon \eta / 2} ; \epsilon k+\nu l+\epsilon \eta\right) \\
G^{a}(z ; k) G^{b}(w ; l)=\frac{1}{4} \sum_{\epsilon, \eta, \nu}(-\epsilon)^{\frac{1-a}{2}}(-\nu)^{\frac{1-b}{2}} \frac{\left(P^{\nu} Q^{\epsilon} q^{-\eta}\right)^{-1 / 2}}{z Q^{\epsilon}-w P^{\nu} q^{\eta}}\left(\frac{1 / 2}{\left(z Q^{-\epsilon}-w P^{-\nu}\right)_{q}^{2}}\right. \\
\left.+T\left(w Q^{-\epsilon} q^{\eta / 2} ; a b \eta \nu l-a b \eta \epsilon k+a b\right)\right) .
\end{gathered}
$$

These results are checked in the similar way as previous section.

Finally, we make a comment on this superalgebra. Although we have introduced two kind of deformed supercurrents (3.3) following to ref. [2], we can simplify the algebra and 
reduce the number of anti-commutation relations among $G^{ \pm}$. Decomposing $G^{ \pm}$into two pieces as

$$
G^{ \pm}(r, k)=\frac{1}{2}(\tilde{G}(r,-k) \pm \tilde{G}(r, k))
$$

we change eq.(3.6) to

$$
\left\{\tilde{G}(r, k), d_{s}\right\}=q^{\left(\frac{r}{2}+s\right) k}[s]_{+} a_{r+s}, \quad\left[\tilde{G}(r, k), a_{m}\right]=-[m]_{+} q^{-\left(\frac{r}{2}+s\right) k} d_{r+m}
$$

Commutation relations among $B, H$ and $\tilde{G}$ are in turn

$$
\begin{gathered}
{[B(n, k), \tilde{G}(r, l)]=G \rightarrow \tilde{G} \text { in }(2.34)} \\
{[H(n, k), \tilde{G}(r, l)]=\frac{1}{2\left(q-q^{-1}\right)} \sum_{\epsilon, \eta} \epsilon q^{\frac{n(l+\eta)-r(\epsilon k+\eta)}{2}} \tilde{G}(n+r, l+\epsilon k+\eta)} \\
\{\tilde{G}(r, k), \tilde{G}(s, l)\}=\sum_{\epsilon}\left(q^{s k+r l+(s-r) \epsilon} B(r+s, k-l+\epsilon)\right. \\
\left.+\epsilon q^{-s k-r l-(s-r) \epsilon} H(r+s, k-l+\epsilon)\right)+\tilde{C}^{G}(r \mid l, k) \delta_{r+s, 0} .
\end{gathered}
$$

Taking account of the properties

$$
B(n, k)=B(n,-k), \quad H(n, k)=-H(n,-k)
$$

we can cast them into the following simple superalgebra

$$
\begin{gathered}
{[L(n, k), \tilde{G}(r, l)]=\frac{1}{\left(q-q^{-1}\right)} \sum_{\epsilon, \eta} \epsilon\left[\frac{n(l+\eta)-r(\epsilon k+\eta)}{2}\right]_{-\epsilon \eta} \tilde{G}(n+r, l+\epsilon k+\eta)} \\
\{\tilde{G}(r, k), \tilde{G}(s, l)\}=\sum_{\epsilon, \eta}\left[\frac{s(k+\epsilon)+r(l-\epsilon)}{2}\right]_{\epsilon \eta} L(r+s, \eta k-\eta l+\epsilon \eta)+\tilde{C}^{G}(r \mid l, k) \delta_{r+s, 0} .
\end{gathered}
$$

Here the number of independent (anti-)commutator brackets in (3.7)-(3.9) reduces to 3 from 6. $L$ and $\tilde{G}$ are now of course not symmetric under $k \rightarrow-k$. OPEs for (3.29) and (3.30) are given by

$$
\begin{gathered}
T(z ; k) \tilde{G}(w ; l)=\frac{1}{2 w\left(q-q^{-1}\right)} \sum_{\epsilon, \nu, \eta} \frac{\epsilon^{\frac{1+\nu}{2}} \eta^{\frac{1-\nu}{2}}\left(Q^{\epsilon} q^{\eta / 2}\right)^{-1 / 2}}{\left(z Q^{-\epsilon}-w P^{\nu} q^{\eta}\right) P^{\nu}} \tilde{G}\left(w Q^{\epsilon} q^{\eta / 2} ; l+\epsilon \nu k+\nu \eta\right) \\
\tilde{G}(z ; k) \tilde{G}(w ; l)=\frac{1}{2} \sum_{\epsilon, \nu, \eta}\left((-\epsilon \eta)^{\frac{1-\nu}{2}} \frac{\left(P^{\nu} Q^{\nu} q^{-\epsilon}\right)^{-1 / 2}}{z Q^{\nu}-w P^{\nu} q^{\epsilon}} T\left(w Q^{-\nu} q^{\epsilon / 2} ; \eta l-\eta k+\epsilon \nu \eta\right)\right. \\
\left.+\frac{\frac{1}{4}\left(P Q q^{-\epsilon}\right)^{-1 / 2}}{\left(z Q-w P q^{\epsilon}\right)\left(z Q^{-1}-w P^{-1}\right)_{q}^{2}}\right) .
\end{gathered}
$$

These are consistent with (3.21) and (3.22) under the relation (3.23). 


\section{Deformation in $b c$ system}

\section{1 deformed algebra $q-\mathrm{Vir}^{b c}$}

Let us consider the following deformed ghost Virasoro operators

$$
L^{b c}(n, k)=\frac{1}{[k]} \sum_{m}[k(n \Delta-n+m)]: b_{n-m} c_{m}:
$$

with the commutation relation [9]

$$
c_{n} b_{m}+\epsilon b_{m} c_{n}=\delta_{n+m, 0}
$$

where $\Delta$ is the conformal weight for $b$ and $\epsilon$ means the statistics $(\Delta=2, \epsilon=1$ for ghosts and $\Delta=3 / 2, \epsilon=-1$ for superghosts) and

$$
b(z)=\sum_{n \in Z-\Delta} b_{n} z^{-n-\Delta}, \quad c(z)=\sum_{n \in Z+\Delta} c_{n} z^{-n-(1-\Delta)} .
$$

Eq.(4.1) satisfies

$$
\left[L^{b c}(n, k), b_{m}\right]=\frac{1}{[k]}[k(n \Delta-n-m)] b_{n+m}, \quad\left[L^{b c}(n, k), c_{m}\right]=\frac{-1}{[k]}[k(n \Delta+m)] c_{n+m} .
$$

If we put $\Delta=1 / 2$, the generators (4.1) are similar to those for $q-\operatorname{Vir}^{F}$ and actually satisfy the same commutation relation as (2.5) with the central term $2 C^{F}$. While for any other values of $\Delta$, we have to introduce an additional set of operators which vanishes in the limit $q \rightarrow 1$ in order that its algebra may be closed. For example, we choose it

$$
O^{b c}(n, k)=\frac{[1]_{-}}{[k]} \sum_{m}[k(n \Delta-n+m)]_{+}: b_{n-m} c_{m}:,
$$

which satisfies

$$
\left[O^{b c}(n, k), b_{m}\right]=\frac{[1]_{-}}{[k]}[k(n \Delta-n-m)]_{+} b_{n+m}, \quad\left[O^{b c}(n, k), c_{m}\right]=\frac{-[1]_{-}}{[k]}[k(n \Delta+m)]_{+} c_{n+m} .
$$

We thereby obtain the following closed algebra as a deformed Virasoro algebra (for later convenience, we call it $q-\mathrm{Vir}^{b c}$ )

$$
\left[L^{b c}(n, k), L^{b c}(m, l)\right]=\sum_{\epsilon= \pm 1} \frac{[(n \epsilon l-m k) / 2][k+\epsilon l]}{[k][\epsilon l]}\left(\left[\left(\frac{1}{2}-\Delta\right)(\epsilon n l+m k)\right]_{+} L^{b c}(n+m, k+\epsilon l)\right.
$$




$$
\begin{gathered}
\left.+\left[\left(\frac{1}{2}-\Delta\right)(\epsilon n l+m k)\right] O^{b c}(n+m, k+\epsilon l) /[1]_{-}\right)+C^{L L}(n \mid k, l) \delta_{n+m, 0} \\
{\left[L^{b c}(n, k), O^{b c}(m, l)\right]=\sum_{\epsilon= \pm 1} \frac{[(n \epsilon l-m k) / 2][k+\epsilon l]}{[k][l]}\left(\left[\left(\frac{1}{2}-\Delta\right)(\epsilon n l+m k)\right]_{+} O^{b c}(n+m, k+\epsilon l)\right.} \\
\left.+[1]_{-}^{2}\left[\left(\frac{1}{2}-\Delta\right)(\epsilon n l+m k)\right]_{-} L^{b c}(n+m, k+\epsilon l)\right)+C^{L O}(n \mid k, l) \delta_{n+m, 0} \\
{\left[O^{b c}(n, k), O^{b c}(m, l)\right]=\sum_{\epsilon= \pm 1} \frac{[(n \epsilon l-m k) / 2][k+\epsilon l]}{[k][l]}\left([1]_{-}^{4}\left[\left(\frac{1}{2}-\Delta\right)(\epsilon n l+m k)\right]_{+} L^{b c}(n+m, k+\epsilon l)\right.} \\
\left.+[1]_{-}^{2}\left[\left(\frac{1}{2}-\Delta\right)(\epsilon n l+m k)\right]_{-} O^{b c}(n+m, k+\epsilon l)\right)+C^{O O}(n \mid k, l) \delta_{n+m, 0}
\end{gathered}
$$

where

$$
\begin{gathered}
C^{L L}(n \mid k, l)=\frac{\epsilon}{2[k][l]}\left(\sum_{0<m \leq n}+\sum_{0 \leq m<n}\right)_{m \in Z-\Delta}[(n \Delta-m) k][(n-m-n \Delta) l], \\
C^{L O}(n \mid k, l)=\frac{\epsilon[1]_{-}}{2[k][l]}\left(\sum_{0<m \leq n}+\sum_{0 \leq m<n}\right)_{m \in Z-\Delta}[(n \Delta-m) k][(n-m-n \Delta) l]_{+}, \\
C^{O O}(n \mid k, l)=\frac{\epsilon[1]_{-}^{2}}{2[k][l]}\left(\sum_{0<m \leq n}+\sum_{0 \leq m<n}\right)_{m \in Z-\Delta}[(n \Delta-m) k]_{+}[(n-m-n \Delta) l]_{+} .
\end{gathered}
$$

Only (4.7) survives in $q \rightarrow 1$ reducing to the usual ghost Virasoro algebra. Note that the center $C^{L L}$ coincides with $2 C_{R}^{F}$ for sum over integers on $m$ as well as with $2 C^{F}$ for half integers after putting $\Delta=1 / 2$ and $\epsilon=1$. This is very similar to the $q=1$ case.

\section{2 realization of $q-\mathrm{Vir}^{F}$}

The set of eqs.(4.1) and (4.5) is composed of a linear combination of two independent (positive/negative) powers of $q$, namely of $\sum q^{m} b_{n-m} c_{m}$ and $\sum q^{-m} b_{n-m} c_{m}$. Recombining these operators we can eliminate the extra operator $O^{b c}$ from (4.7), and we thus obtain a closed algebra for single generator. To see this, let us consider the following operators as a recombination:

$$
\begin{aligned}
& F^{b c}(n, k)=\frac{-1}{[k]} \sum_{m}\left[k\left(\frac{n}{2}-m\right)\right]: b_{n-m} c_{m}: \\
& R^{b c}(n, k)=\frac{1}{[k]} \sum_{m}\left[k\left(\frac{n}{2}-m\right)\right]_{+}: b_{n-m} c_{m}:
\end{aligned}
$$


which satisfies

$$
\begin{gathered}
{\left[F^{b c}(n, k), b_{m}\right]=\frac{-1}{[k]}\left[k\left(\frac{n}{2}+m\right)\right] b_{n+m}, \quad\left[R^{b c}(n, k), b_{m}\right]=\frac{1}{[k]}\left[k\left(\frac{n}{2}+m\right)\right]_{+} b_{n+m}} \\
{\left[F^{b c}(n, k), c_{m}\right]=\frac{1}{[k]}\left[k\left(\frac{n}{2}+m\right)\right] c_{n+m}, \quad\left[R^{b c}(n, k), c_{m}\right]=\frac{-1}{[k]}\left[k\left(\frac{n}{2}+m\right)\right]_{+} c_{n+m} .}
\end{gathered}
$$

With these formulae, we obtain the following results

$$
\begin{aligned}
& {\left[F^{b c}(n, k), F^{b c}(m, l)\right]=\sum_{\epsilon= \pm 1} \frac{[(n \epsilon l-m k) / 2][k+\epsilon l]}{[k][\epsilon l]} F^{b c}(n+m, k+\epsilon l)+C^{F F}(n \mid k, l) \delta_{n+m, 0}} \\
& {\left[F^{b c}(n, k), R^{b c}(m, l)\right]=\sum_{\epsilon= \pm 1} \frac{[(n \epsilon l-m k) / 2][k+\epsilon l]}{[k][l]} R^{b c}(n+m, k+\epsilon l)+C^{F R}(n \mid k, l) \delta_{n+m, 0}} \\
& {\left[R^{b c}(n, k), R^{b c}(m, l)\right]=[1]_{-}^{2} \sum_{\epsilon= \pm 1} \frac{[(n \epsilon l-m k) / 2][k+\epsilon l]}{[k][l]} F^{b c}(n+m, k+\epsilon l)+C^{R R}(n \mid k, l) \delta_{n+m, 0},}
\end{aligned}
$$

where

$$
\begin{gathered}
C^{F F}(n \mid k, l)=\frac{\epsilon}{[k][l]} \sum_{0<m \leq n, m \in Z-\Delta}\left[\left(\frac{n}{2}-m\right) k\right]\left[\left(\frac{n}{2}-m\right) l\right] \\
C^{F R}(n \mid k, l)=\frac{\epsilon}{[k][l]} \sum_{0<m \leq n, m \in Z-\Delta}\left[\left(\frac{n}{2}-m\right) k\right]\left[\left(\frac{n}{2}-m\right) l\right]_{+} \\
C^{R R}(n \mid k, l)=\frac{\epsilon}{[k][l]} \sum_{0<m \leq n, m \in Z-\Delta}\left[\left(\frac{n}{2}-m\right) k\right]_{+}\left[\left(\frac{n}{2}-m\right) l\right]_{+} .
\end{gathered}
$$

We thereby note that eq.(4.13) forms a closed algebra by itself and the algebra (4.16) coincides with the $q-\mathrm{Vir}^{F}$ commutation relation. We can construct the OPE formula also for this ghost realization of $q$-Vir ${ }^{F}$ in the following way. Defining field corresponding to $F^{b c}(n, k)$

$$
F^{b c}(z ; k)=\sum_{n} F^{b c}(n, k) z^{-n-2}=\frac{-1}{[k]\left(q-q^{-1}\right) z}\left(q^{k(1-2 \Delta) / 2} b\left(z Q^{-1}\right) c(z Q)-\left(q \rightarrow q^{-1}\right)\right)
$$

and using

$$
\begin{aligned}
F^{b c}(z ; k) b(w) & =\frac{-1}{[k]\left(q-q^{-1}\right) w}\left(Q^{2-2 \Delta} \frac{b\left(w Q^{-2}\right)}{z Q-w}-\left(q \rightarrow q^{-1}\right)\right) \\
F^{b c}(z ; k) c(w) & =\frac{1}{[k]\left(q-q^{-1}\right) w}\left(Q^{-2 \Delta} \frac{c\left(w Q^{2}\right)}{z Q^{-1}-w}-\left(q \rightarrow q^{-1}\right)\right),
\end{aligned}
$$


we obtain the OPE corresponding to (4.16)

$$
\begin{aligned}
F^{b c}(z ; k) F^{b c}(w ; l) & =\frac{\epsilon}{[k][l]\left(q-q^{-1}\right)^{2} z w} \sum_{\epsilon, \nu} \frac{\epsilon \nu\left(Q^{\epsilon} P^{\nu}\right)^{1-2 \Delta}}{\left(z Q^{-\epsilon}-w P^{\nu}\right)\left(z Q^{\epsilon}-w P^{-\nu}\right)} \\
& +\frac{1}{w\left(q-q^{-1}\right)} \sum_{\epsilon, \eta= \pm 1} \frac{[\epsilon k+\eta l]}{[\epsilon k][\eta l]} \frac{P^{-\eta}}{z Q^{-\epsilon}-w P^{\eta}} F^{b c}\left(w Q^{\epsilon} ; \epsilon k+\eta l\right) .
\end{aligned}
$$

This is same as (2.31) up to difference between central terms.

One may expect to construct a superalgebra for $q$-Vir ${ }^{b c}$ or $q$ - $\mathrm{Vir}^{F}$ in terms of $b c$ and $\beta \gamma$ ghosts. Unfortunately we have not succeeded in constructing a desirable superalgebra which reduces to the conventional Virasoro superalgebra. We here only present a basic closed superalgebra, which might produce some kind of deformed Virasoro superalgebra through recombination. Introducing $\beta \gamma$ fields with conformal dimensions $\lambda$ in addition to 4.13 ) and (4.14), we can verify that

$$
\begin{aligned}
& F^{\beta \gamma}(n, k)=\frac{-1}{[k]} \sum_{m \in Z+\lambda}\left[k\left(\frac{n}{2}-m\right)\right]: \beta_{n-m} \gamma_{m}: \\
& R^{\beta \gamma}(n, k)=\frac{1}{[k]} \sum_{m \in Z+\lambda}\left[k\left(\frac{n}{2}-m\right)\right]_{+}: \beta_{n-m} \gamma_{m}:
\end{aligned}
$$

and

$$
J^{ \pm}(n, k)=\sum_{m \in Z-\Delta} q^{k(m-n / 2)}: b_{m} \gamma_{n-m}: \pm \sum_{m \in Z+\Delta} q^{k(m-n / 2)}: c_{m} \beta_{n-m}:
$$

satisfy the following closed algebra

$$
\begin{gathered}
{\left[F^{b c}(n, k), J^{ \pm}(m, l)\right]=\frac{1}{2[k][1]_{-}}\left(-q^{\frac{m k-n l}{2}} J^{ \pm}(n+m, l+k)+q^{\frac{-m k-n l}{2}} J^{ \pm}(n+m, l-k)\right)} \\
{\left[F^{\beta \gamma}(n, k), J^{ \pm}(m, l)\right]=\frac{1}{2[k][1]_{-}}\left(q^{\frac{-m k+n l}{2}} J^{ \pm}(n+m, l+k)-q^{\frac{m k+n l}{2}} J^{ \pm}(n+m, l-k)\right)} \\
{\left[R^{b c}(n, k), J^{ \pm}(m, l)\right]=\frac{1}{2[k]}\left(q^{\frac{m k-n l}{2}} J^{\mp}(n+m, l+k)+q^{\frac{-m k-n l}{2}} J^{\mp}(n+m, l-k)\right)} \\
{\left[R^{\beta \gamma}(n, k), J^{ \pm}(m, l)\right]=\frac{1}{2[k]}\left(-q^{\frac{-m k+n l}{2}} J^{\mp}(n+m, l+k)-q^{\frac{m k+n l}{2}} J^{\mp}(n+m, l-k)\right)} \\
\left\{J^{ \pm}(n, k), J^{ \pm}(m, l)\right\}= \pm 2[k-l]\left(q^{\frac{m k+n l}{2}} R^{b c}(n+m, k-l)+q^{-\frac{m k+n l}{2}} R^{\beta \gamma}(n+m, k-l)\right)
\end{gathered}
$$




$$
\begin{aligned}
& \pm\left(q-q^{-1}\right) \frac{[n(k-l) / 2]}{[(k-l) / 2]}[(k-l) f(\Delta)] \delta_{n+m, 0} \\
\left\{J^{ \pm}(n, k), J^{\mp}(m, l)\right\}= & \pm 2[k-l][1]_{-}\left(q^{\frac{m k+n l}{2}} F^{b c}(n+m, k-l)+q^{-\frac{m k+n l}{2}} F^{\beta \gamma}(n+m, k-l)\right) \\
\mp & 2 \frac{[n(k-l) / 2]}{[(k-l) / 2]}\left[(k-l)\left(\Delta-[\Delta]_{G}-\frac{1}{2}\right)\right]_{+} \delta_{n+m, 0}
\end{aligned}
$$

where

$$
f(\Delta)= \begin{cases}0, & \text { for integral } \Delta \\ \Delta-[\Delta]_{G}-1 / 2, & \text { for rational } \Delta\end{cases}
$$

and []$_{G}$ means the Gauss bracket.

\section{Conclusion}

We have calculated the OPE formulae for three types of the deformed super-Virasoro algebras and considered a construction of deformed Virasoro algebra in terms of ghost Heisenberg oscillators. The most important problem is that the deformed Virasoro algebra discussed here in $b c$ system has different structure constants from those of $q$-Vir ${ }^{F}$ or $q$-Vir ${ }^{B}$ and we do not know which deformed Virasoro algebra should be shared by bosonic, fermionic and ghost realizations. If the $q-\mathrm{Vir}^{B} / q-\mathrm{Vir}^{F}$ is a common algebra, we have to find its realization in terms of $b c /$ bosonic system. If $q$ - $\mathrm{Vir}^{b c}$, we must find both bosonic and fermionic realizations. We may have to examine various possibility including generalized algebras [11] or standing on a more fundamental basis like in [3], [4], [5] to find an appropriate unique algebra.

Although the determination of common structure constants is necessary for supersymmetric argument and cancellation of anomalies etc., we can demonstrate how the critical dimensions may depend on the deformation parameter $q$ supposing only fermionic and ghost realization of $q$ - $\operatorname{Vir}^{F}$. The anomaly free equation for fermion-ghost $q$ - $\operatorname{Vir}^{F}$ operator $L(n, k)=F(n, k)+F^{b c}(n, k)-a(k) \delta_{n, 0}$, where $a(k)$ is a shift of zero mode, leads us to a relation between $a(k)$ and the number of fermion $D$;

$$
a(k)=\frac{-\left(2 D+q^{k / 2}+q^{-k / 2}\right)}{[k][k / 2]\left(q-q^{-1}\right)^{2}} .
$$


This allows that $D$ might vary depending on the value of $q$. Note that the $q$ - $\operatorname{Vir}^{F}$ in $b c$ system certainly satisfies the Virasoro algebra in $q \rightarrow 1$, however this limit is meaningless because there is no corresponding field operator in $q=1$ as can be easily seen from (4.22). While the $q \neq 1$ case, we can expand $F^{b c}(n, k)$ into an infinite sum of operators with various conformal weights, and we hence regard it as a kind of $W_{1+\infty}$ algebra [12]. Nevertheless to find a similar equation as above is interesting in the context of deforming string theories.

All of the deformed algebras discussed here belong not to the quantum groups but to the Lie algebras. If we want to construct a deformed string theory identifying its worldsheet with quantum plane which is a basis of quantum groups, we must deal with a field theory on quantum plane. To extract the Fourier mode operators of the Virasoro type constraints from quantum plane, we need a contour integral on quantum plane, however it has not been defined yet. At present, it is natural to consider that our deformation might be a profile projected from strings on quantum spacetime. The relationship between quantum groups and our deformed Virasoro algebras should be clarified in future study.

There are some unsolved problems suggested above, however we expect that our analysis presented here may provide interesting and useful information for integrable models, string and conformal field theories as well as for application to quantum groups and $W$-infinity symmetries [13].

\section{Acknowledgements}

The author would like to thank T. Kobayashi for discussion and N. Aizawa, S. Lukyanov and S. Odake for useful communication. 


\section{A}

All commutation relations between generators and fields for Chaichian-Presnajder's superalgebra are summarized.

$$
\begin{gathered}
\{G(r, k), \psi(z)\}=z^{r+1 / 2} q^{-k(z \partial+r / 2+1)} \Phi(z) \\
{[F(n, k), \psi(z)]=z^{n} \frac{1}{[k]}\left[k\left(z \partial+\frac{1}{2}\right)+n k / 2\right] \psi(z)} \\
{[G(r, k), \Phi(z)]=z^{r-1 / 2} q^{k z \partial+k(r+1) / 2}\left[z \partial+r+\frac{1}{2}\right] \psi(z)} \\
{[B(n, k), \Phi(z)]=z^{n} \sum_{\eta= \pm 1} \frac{\eta}{2}\left[(k+\eta)(z \partial+1)+\frac{n}{2}(k+2)\right] \Phi(z)} \\
{[F(n, k), G(z ; l)]=\frac{z^{n}}{[k]\left(q-q^{-1}\right)} q^{n l / 2} \sum_{\epsilon= \pm 1} \epsilon q^{\epsilon k(z \partial+3 / 2+n) / 2} G(z ; l+\epsilon k)} \\
\{B(n, k), G(z ; l)]=\frac{z^{n}}{2\left(q-q^{-1}\right)} q^{-n l / 2} \sum_{\epsilon, \eta= \pm 1} \eta q^{\epsilon k(z \partial+3 / 2+n) / 2} q^{\eta\left(z \partial+\frac{3}{2}+2 n\right) / 2} G(z ; \epsilon k-l+\eta) \\
\{G(r, k), G(z ; l)\}=C^{G}(r \mid l, k) z^{r-3 / 2}+2 z^{r+1 / 2} q^{r l / 2} q^{-k(z \partial+r+2) / 2} B(z ; k-l) \\
{[B(n, k), B(z ; l)]=z^{r+1 / 2} q^{-r l / 2} q^{k(z \partial+r+2) / 2} \sum_{\epsilon} \epsilon q^{\epsilon k(z \partial+2 r+2) / 2}[k-l+\epsilon] F(z ; k-l+\epsilon)} \\
{[F(n, k), F(z ; l)]=C^{F}(n \mid k, l) z^{n-2}+z^{n} \sum_{\epsilon, \eta} \frac{\eta}{2}\left[\frac{1}{2}(k+\eta)(z \partial+2)+\frac{n}{2}(k+\epsilon l+2 \eta)\right] B(z ; k+\epsilon l+\eta)} \\
{\left[\begin{array}{l}
{[\text { A }} \\
{[(k)}
\end{array}\right.}
\end{gathered}
$$

\section{References}

[1] M.Chaichian and P.Presnajder, Phys. Lett. B277 (1992) 109.

[2] A.A. Belov and K.D. Chaltikian, Mod. Phys. Lett. A8 (1993) 1233.

[3] S. Lukyanov and Y. Pugai, "Bosonization of ZF algebras: direction toward deformed Virasoro algebra", hep-th/9412128. 
[4] E. Frenkel and N. Reshetikhin, "Quantum affine algebras and deformations of the Virasoro and W-algebras", q-alg/9505025.

[5] J. Shiraishi, H. Kubo, H. Awata and S. Odake, "A quantum deformation of the Virasoro algebra and the Macdonald symmetric functions", q-alg/9507034.

[6] S. Lukyanov, "A note on the deformed Virasoro algebra", hep-th/9509037.

[7] H. Sato, Nucl. Phys. B393 (1993) 442.

[8] C.H. Oh and K. Singh, "Realizations of the q-Heisenberg and q-Virasoro Algebras", hep-th/9408001.

[9] D. Friedan, E. Martinec and S. Shenker, Nucl. Phys. B271 (1986)93.

[10] H. Hiro-oka, O. Matsui, T. Naito and S. Saito, TMUP-HEL-9004 (1990).

[11] R. Kemmoku and S. Saito, Phys. Lett. B319 (1993) 471.

[12] R. Kemmoku and S. Saito, " $W_{1+\infty}$ as a discretization of Virasoro algebra", hepth/9411027.

[13] H. Sato, Mod. Phys. Lett. A9 (1994) 451; ibid. 1819; Prog. Theor. Phys. 93 (1995) 195. 